\section{Estudio de los conocimientos, actitudes y prácticas de la leishmaniasis: evidencias del olvido estatal en el Darién Colombiano}

\author{
Study of knowledge, attitudes, and practices \\ related to leishmaniasis: evidence of government \\ neglect in the Colombian Darién
}

\section{Estudo dos conhecimentos, atitudes e práticas da leishmaniose: evidências da negligência do Estado no Darién colombiano}

\begin{abstract}
1 Programa de Estudio y Control de Enfermedades Tropicales, Universidad de Antioquia, Medellín, Colombia.

${ }^{2}$ Facultad de Ciencias Agrarias, Universidad de Antioquia, Medellín, Colombia.

3 Grupo Medio Ambiente y Sociedad, Universidad de Antioquia, Medellín, Colombia.

Correspondencia L. M. Carrillo-Bonilla Programa de Estudio y Control de Enfermedades Tropicales, Universidad de Antioquia.

Calle 70 No 52-21, Medellín, Medellín - 0000, Colombia. linacarrillo@gmail.com
\end{abstract}

\begin{abstract}
American cutaneous leishmaniasis (ACL) is considered a public health problem worldwide, and incidence in Colombia is 12,000 cases per year. Colombia has implemented control programs for years which have often overlooked the social, economic, and demographic characteristics of the regions where the disease occurs. As part of an epidemiological study, this article presents the results of a survey on knowledge, attitudes, and practices (KAP) related to leishmaniasis in rural populations in Acandi in the Colombian Darién. Qualitative and quantitative tools were used (ethnography and surveys, respectively). The results show scarce knowledge among the population on basic aspects such as recognition of the sand fly vector. There were also differences in KAP according to gender and type of population, rather than by geographic area. The study points to government neglect as a critical factor in the persistence of the disease.
\end{abstract}

Cutaneous Leishmaniasis; Health Knowledge, Attitudes, Practice; Neglected Diseases
Lina María Carrillo-Bonilla 1,2 Juan José Trujillo 1 Lizeth Álvarez-Salas 3 Iván Darío Vélez-Bernal 1

\section{Resumen}

La leishmaniasis cutánea americana (LCA) es considerada un problema de salud pública a nivel mundial, y su incidencia en Colombia es de 12 mil casos por año. Durante años se han implementado programas de control, que frecuentemente ignoran características sociales, económicas y demográficas de las regiones afectadas. Como parte de un estudio de tipo epidemiológico, en este artículo se presentarán los resultados de un estudio sobre los conocimientos, actitudes y prácticas (CAP) frente a la leishmaniasis, que tienen las poblaciones de las zonas rurales del municipio de Acandí, en el Darién Colombiano. Para el estudio se utilizaron herramientas cualitativas como la etnografía y cuantitativas, como las encuestas dirigidas. Los resultados ponen en manifiesto el desconocimiento de la población en aspectos fundamentales como el reconocimiento del vector. Además, existen diferencias en las CAP por género y por tipo de población, pero no por su distribución geográfica. Se evidenció el abandono estatal como factor determinante en la perpetuación de la enfermedad.

Leishmaniasis Cutánea; Conocimientos, Actitudes y Práctica en Salud; Enfermedades Desatendidas 


\section{Introducción}

Los desafíos planteados por las enfermedades tropicales son enormes, tan grandes como sus devastadoras consecuencias; 655 mil muertos por malaria y 2 millones de enfermos por leishmaniasis para el año 2012 son algunos de los datos que lo demuestran 1. La leishmaniasis americana es causada por parásitos del género Leishmania y trasmitida por flebótomos del género Lutzomyia. Tiene tres formas clínicas, visceral (LV), cutánea (LC) y mucocutánea (LMC). La forma cutánea, que es la más prevalente en Colombia, se caracteriza por la presencia de úlceras indoloras en el sitio de la picadura del insecto vector, que pueden permanecer de manera crónica durante años 2 . En este país la enfermedad se distribuye por todo el territorio, y aunque se acepta un considerable subregistro, hay un claro aumento de su incidencia, pasando de tener en promedio 6 mil casos por año en la década de los 90, a más de 12 mil aproximadamente en la última década 3 .

Este aumento en el número de casos, no sólo en leishmaniasis, sino en otras enfermedades tropicales como la malaria y dengue, demuestra el fracaso en los programas para su control. Tal vez, una razón para este fenómeno es la forma como han sido concebidas las enfermedades tropicales y analizadas desde sólo su dimensión biológica, cuyas investigaciones epidemiológicas tienen un enfoque exclusivo sobre los factores de riesgo a nivel individual dentro de la población, denominado el paradigma de la caja negra en la epidemiología 4 . El estudio de las enfermedades tropicales como la leishmaniasis ha sido en muchos casos reduccionista y ha particularizando el riesgo de enfermar. Se ha desconocido el fuerte componente socioeconómico y cultural que determina su presencia y dispersión. A su vez, los estudios de la enfermedad no han dimensionado los efectos que las políticas públicas podrían generar en lugares con escasa presencia estatal. Es posible inferir que aún se desconoce el complejo entramado que facilita la presentación y distribución de estas dolencias, que podrían ser develadas si las investigaciones tuvieran una visión holística del fenómeno.

La inclusión de un enfoque metodológico interdisciplinar, que permita la participación de diferentes áreas del conocimiento para el abordaje del estudio de estas enfermedades, es fundamental. Esto implica integrar de manera sistemática el componente cualitativo a los estudios epidemiológicos, ya sea desde la sociología y/o la antropología 5. Esta conjunción ha venido dándose en América Latina de forma aislada desde principios del siglo XX, y un poco más organizada después de los años 60, sin embargo falta mucho por construir 6 . Dentro del estudio de los factores sociales determinantes en salud, está el estudio de los conocimientos, actitudes y prácticas (CAP). En ellas se incluye el sistema de creencias acerca de las causas y el significado de la enfermedad, los sistemas médicos, esto es los procedimientos para diagnosticarla, tratarla y prevenirla.

El presente artículo presenta los resultados de un estudio cualitativo y cuantitativo sobre los CAP, frente a la leishmaniasis que tienen algunas poblaciones del municipio de Acandí (Chocó), Colombia, realizado en el marco del proyecto Estudio Ecoepidemiológico para la Prevención y el Control de la Leishmaniasis en Acandí, Chocó.

\section{Situación actual del Chocó}

Durante la conquista española en América, la reducción en el número de indígenas, obligó a los españoles a importar esclavos africanos para trabajar en las minas del Chocó. Con el tiempo los esclavos pasaron a ser el grupo más abundante en la región. Desde el comienzo, las duras condiciones climatológicas de la zona, clima caliente y húmedo, donde prosperaban las enfermedades tropicales marcaban su desarrollo social. La población chocoana se encontraba dispersa, y geográficamente aislada, con propietarios ausentes, predominio de la economía basada en un solo producto: el oro. Posteriormente, el aislamiento geográfico, la escasa presencia del estado, la fragilidad institucional y la explotación sin control de los recursos naturales, han provocado un bajo nivel de desarrollo en la región. Este fenómeno es evidente en los bajos niveles de escolaridad, baja cobertura en salud y altos índices de pobreza 7 .

El Chocó tiene el nivel más bajo de kilómetros de carretera pavimentada por cada mil habitantes. El porcentaje de analfabetismo es el doble del promedio nacional. De acuerdo a la base de datos de Scopus, mientras para el país se produjeron 4.859 manuscritos científicos en el año 2010, sólo seis tuvieron filiación con instituciones del departamento del Chocó 8,9.

Las cifras en salud son menos esperanzadoras. El $30 \%$ de la población no se encuentra afiliado en ningún régimen de salud contributivo o subsidiado. Los servicios como conducciones de agua y alcantarillado, que tienen una influencia directa sobre las condiciones de salud, sólo alcanzan coberturas del 22 al 25\% respectivamente 10 . Según la Secretaría de Salud Departamental del Chocó, para el año 2008 se presentaron 2.511 casos de desnutrición infantil y cada mes mueren al menos 17 niños por esta causa 10 . 
Las altas tasas de morbilidad y mortalidad de enfermedades infecciosas se encuentran estrechamente ligadas a la pobreza. La leishmaniasis, de hecho, ha sido relacionada con los bajos ingresos económicos (de hasta menos de 1 dólar por día en algunos países), pues la pobreza a su vez se encuentra asociada a factores sociales, políticos y económicos que aumentan el riesgo de la enfermedad, tales como mala disposición de excretas, deficientes condiciones de vivienda, malnutrición, pobres esquemas de control vectorial, etc. ${ }^{11}$. Se ha evidenciado, por ejemplo, que una baja ingesta de proteínas aumenta el riesgo de adquirir LV 12.

No es de extrañar que una de las regiones más abandonadas de un país subdesarrollado, y que además presenta todas las condiciones necesarias para que proliferen los vectores y se mantenga la transmisión, presente altos índices de incidencia de enfermedades como malaria y leishmaniasis. Sin embargo, para el año 2008 el sistema de vigilancia epidemiológica nacional (Sistema de Vigilancia en Salud Pública SIVIGILA) reportó para el departamento del Chocó tan solo 18 casos de LC, equivalente al 0,54\% de los casos informados para el país en ese año, lo que tampoco debe extrañar, pues la deficiencia en las coberturas de salud se acompañan de altísimos subregistros 13 .

\section{Metodología}

Este trabajo se llevó a cabo durante un año entre noviembre del 2008 al 2009 en el municipio de Acandí del departamento de Chocó, Colombia, en los corregimientos de San Francisco de Asís, Triganá y Capurganá (veredas El Aguacate y La Mora), localizadas en la zona costera. Y en Santa Cruz de Chugandí, localizado en estribaciones de la serranía de Tripogadí.

San Francisco es el área con mayor desarrollo urbano, y junto a Triganá y Capurganá, son zonas turísticas, mientras Chugandí es una zona con actividad económica basada en la ganadería y la agricultura de pan coger. Por su aislamiento de la zona costera y centros urbanos importantes, es la localidad más deprimida.

En estas localidades se encuentran asentados cuatro grupos humanos con diferentes orígenes migratorios:

- Población afrodescendiente: originaria de los departamentos de Bolívar, Córdoba y la región del alto Atrato. Esta población está organizada en concejos comunitarios y son poseedores de títulos colectivos de tenencia de tierras localizadas en la serranía del Darién.
- Chilapos: nombre con que se conocen todos aquellos campesinos mestizos, procedentes de los departamentos de Córdoba y Sucre, que migraron de sus tierras para dedicarse a la agricultura 14 .

- Paisas: individuos provenientes de diferentes regiones del país, principalmente del departamento de Antioquia.

- Amerindios: Embera y Embera katio, con una muy baja representatividad y ocupando zonas de resguardos que no fueron incluidos en este estudio.

Los criterios principales para la selección de la zona y la población fueron el número de casos detectados de leishmaniasis y la presencia del vector. Por otro lado, se estudió si las CAP que ha elaborado la comunidad con respecto a la enfermedad son propias y se relacionan con su aislamiento geográfico o, si por el contrario, fueron adquiridas desde afuera y adoptadas por la comunidad.

\section{Estudio de conocimientos, actitudes y prácticas}

Con el fin de identificar las CAP, se implementó una investigación con metodologías tanto cualitativas, como cuantitativas. La etnográfica fue el método cualitativo básico utilizado; en ella se aplicaron las técnicas de observación participante que requiere la convivencia del investigador con la comunidad, donde se posibilitó aprender sobre las costumbres, las formas de ser y las formas de relación con su realidad, las cuales fueron registradas día a día en un diario de campo; posteriormente, los hallazgos fueron analizados y cotejados bibliográficamente. Se realizaron reuniones con la comunidad para confrontar la información entre ellos mismos; se registraron entrevistas semiestructuradas y entrevistas en profundidad con interlocutores claves, los cuales fueron divididos en tres grupos: (1) personas que sufrieron la enfermedad, cuyo acceso a la vivienda fuera posible; (2) personas como curanderos, médicos y enfermeras a los cuales acuden los enfermos y (3) líderes reconocidos de la comunidad: personal de salud local, madres comunitarias, coordinadores de Juntas de Acción Comunal (grupos de agremiación civil) y personal docente de las escuelas y los colegios.

Teniendo en cuenta la información recogida en el trabajo de campo etnográfico, se diseñó una encuesta de 11 preguntas cerradas (Figura 1) sobre los conocimientos, por ejemplo, si conoce la forma de trasmisión de la enfermedad, los síntomas y estrategias de curación, las actitudes y las prácticas como estrategias de control, formas locales de tratamiento de la enfermedad entre 
Figure 1

Encuesta realizada a la comunidad en la zona de estudio.

\author{
1. ¿Qué conoce usted como pito? \\ A. Una enfermedad en forma de llagas \\ B. Un animal (gusano o insecto) \\ C. Una enfermedad transmitida por un mosquito \\ D. No sabe/No responde \\ E. Otro. ¿Cuál? \\ 2. ¿Con cuál otro nombre conoce al pito? \\ A. Yateví \\ B. Bejuco \\ C. Malteveo \\ D. Leishmaniasis \\ 3. ¿Qué conoce usted como bejuco? \\ A. Una planta \\ B. Una enfermedad \\ C. Un mosquito \\ D. Un chinche
}

4. ¿Cómo se transmite la enfermedad?

A. Por la picadura de un mosquito

B. Por un bejuco

C. Por la picadura de una conchilla

D. No sabe/No responde

E. Otro. ¿Cuál?

5. En caso de que la respuesta anterior sea un mosquito, ¿cómo llama al mosquito?

A. Chitra

B. Alus

C. Aludo

D. Jején

E. Palomilla

F. No tiene nombre

G. Otro. ¿Cuál?
7. ¿Cómo se trata la enfermedad?

A. Quemadura con cuchara u otro metal caliente

B. Plantas: ¿cuál?

C. Sustancias químicas: ¿cuál?

D. Pomadas

E. Inyecciones

8. ¿Dónde se contrae la enfermedad?
A. En el bosque
B. En la playa
C. En la casa
D. En la selva
E. Otro. ¿Cuál?

9. ¿Cómo se puede prevenir?

A. Usando ropas largas

B. Usando toldillos

C. Con repelentes

D. Eliminando los mosquitos

E. Gritando yateví

F. No se puede prevenir

G. No sabe/No responde

10. ¿A quién acude cuando está enfermo?

A. Yerbatero

B. Hospital

C. Mayores de edad

D. Usted mismo se trata

E. Otro. ¿Cuál?

11. Cuando algún miembro de la familia se enferma ¿quién se encarga de buscar atención?

A. El papá

B. La mamá

C. El abuelo

D. La abuela

E. El hermano mayor

F. La hermana mayor

G. Otro. ¿Cuál?

6. ¿Cómo sabe usted que alguien tiene pito? (cómo se presenta la enfermedad)

A. Ampolla

B. Grano

C. Callo o verruga

D. Herida/Llaga

E. Roncha

F. No tiene nombre

G. Otro. ¿Cuál? 
otras. Las encuestas se realizaron casa a casa en toda la zona de estudio, previo aviso a líderes y a la comunidad en general. Se encuestó a las personas mayores de 15 años que se encontraban en la residencia en el momento de hacer la visita. Se explicó a las personas de qué se trataba la encuesta y se formulaban las preguntas de forma individual para que los hombres adultos no influenciaran con sus respuestas a las mujeres y a los jóvenes. También se indagó por los papeles que desempeñan hombres y mujeres frente a la enfermedad, con el fin de tener una aproximación cuantitativa sobre las CAP en la región.

La información de campo fue sistematizada y organizada en las categorías de análisis y, posteriormente, fueron procesadas con el programa SPSS 18.0 (IBM Corp., Armonk, Estados Unidos) para identificar asociaciones entre las diferentes categorías de respuestas de las variables sexo y procedencia. Se aplicó la prueba de significancia estadística $\chi^{2}$ y se consideró como significativo un valor de $\mathrm{p}<0,05$.

\section{Comité de ética}

El proyecto Estudio Ecoepidemiológico para la Prevención y el Control de la Leishmaniasis en Acandí, Chocó contó con el aval de la Secretaría de Salud de Chocó (convenio del 16 de marzo de 2009). También fue aprobado por del comité científico de la Sede de Investigación Universitaria de la Universidad de Antioquia, y fue avalado por del comité de ética animal (acta no 38/2007) de la misma universidad. Adicionalmente, el Programa de Estudio y Control de Enfermedades Tropicales (PECET), cuenta con habilitación ante la Dirección de Salud de Antioquia (DSA) para la prestación de servicios de atención en salud y certificación ISO 9001 para la atención de pacientes desde el año 2011.

\section{Resultados y discusión}

Se encuestaron en total 252 personas, 130 hombres y 122 mujeres, todas mayores de 15 años, previo consentimiento de los padres en el caso de los menores de edad (Figura 2).

Las veredas cuentan con una población aproximada de 300 familias ( 1.500 personas), con un alto porcentaje de niños menores de 10 años. Según los datos oficiales del municipio, hay entre cuatro a cinco niños por familia y el $15 \%$ de la población es menor de cinco años 10 .

En general, cada vereda está conformada por dos o tres parentelas que tienen presencia desde su fundación. Además, cohabitan un número significativo de población llegada desde interior del país (zona andina), especialmente desde el departamento de Antioquia y se han mantenido en procesos de migración discontinuos desde hace 40 años. Los primeros colonos llegaron motivados por la posibilidad de acceder a las tierras baldías (son baldíos, y en tal concepto "pertenecen al Estado, los terrenos situados dentro de los límites del territorio nacional que carecen de otro dueño" Art 44, Ley de Tierras 1939), lo que incentivó la migración de más población, que querían adquirir tierras baratas donde asentarse y cultivar en ellas frutales, maderables y productos de pan coger.

La agricultura, la pesca y, en menor medida, la ganadería eran hasta hace poco la base del sustento económico. Sin embargo, la acogida que ha tenido el turismo y especialmente el ecoturismo y el turismo de aventura de bajo costo, influenció hasta mediados de la década del 2000 la trasformación de la economía del lugar.

En una investigación reciente 15 , se indican las nuevas trasformaciones de carácter social y económico que ocurrieron en el último lustro por la presencia permanente de grupos al margen de la ley que ha llevado a un detrimento aún más significativo de la agricultura local, que es ejercida de forma permanente por sólo seis de las 300 familias. Asimismo, se constata la alta tasa de deforestación por la explotación ilegal de la madera, los cultivos ilícitos y la expansión de la ganadería; el deterioro de los recursos marinocosteros que ha generado a su vez la disminución en la pesca y en el consumo de productos marinos; la reducción significativa en la demanda hotelera y la alta dependencia del mercado externo de alimentos y materiales para la construcción, provenientes desde el margen oriental del golfo de Urabá, específicamente desde el Municipio de Turbo (Antioquia). Finalmente, se observa un evidente crecimiento de la población por el efecto de las nuevas migraciones y, por lo tanto, una mayor presión sobre los recursos naturales 16 .

\section{Sobre la enfermedad}

La leishmaniasis es una enfermedad conocida en la región desde hace unos 30 años con el nombre de "pito" o "malteveo" y algunas personas la reconocen como "leishmaniasis". Los más viejos de la comunidad conservan en su memoria otras formas de nombrar este padecimiento como "bejuco" o "yateví", el primero más usado entre los hombres y el segundo entre las mujeres, en una proporción de 13 a 1 .

En cuanto a las denominaciones por vereda, se encontró diferencia significativa para Chugandí, en donde predomina el nombre de "Bejuco" y “Malteveo", pero no "Yatevi”. En Triganá y San 
Figure 2

Distribución porcentual por edades y sexo de la población encuestada en la zona de estudio.

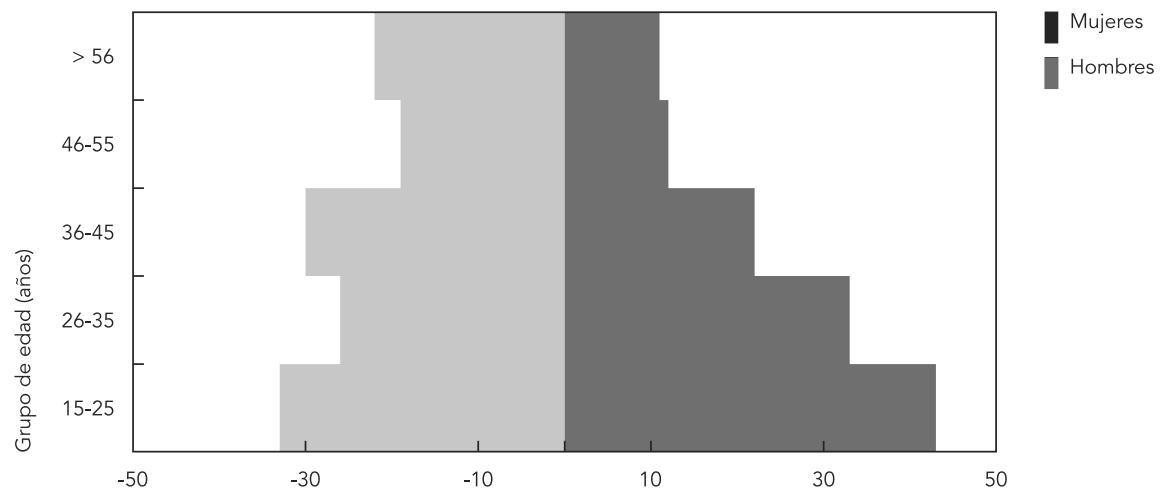

Francisco no relacionan el nombre de "pito" con leishmaniasis. Estos apelativos, que se han encontrado en la costa pacífica colombiana de este Departamento 17 , son representaciones elaboradas a partir del imaginario colectivo para relacionarse con un fenómeno que es desconocido, pero que se pretende hacerlo familiar a partir de creencias y prácticas de la comunidad con respecto a la misma.

"Vos vas caminando en el monte, vas andando y hay una planta que es una especie de palmita que se empieza a mover, el resto está quieto y la matica se está moviendo, le parece a uno casual y sigue caminado y vuelve y aparece, uno se empieza a intrigar como estoy dando vueltas en círculo, cambia su rumbo, cambia el camino y lo hace perder, si le dice "yatevì" se queda quieta y no jode más. Es un espíritu burlón de la selva" (Hombre de 50 años edad, residente en Chugandí).

Umberto Eco se refiere al significado de un término como una unidad que culturalmente está definida y distinguida como entidad por todo el colectivo. Entender estas unidades culturales equivale a entender el lenguaje como un fenómeno social 18. Es así como la comunidad hace referencia a dos tipos de lesiones que nombran y les dan un significado, según la manifestación clínica de la enfermedad, "pito macho" o "pito hembra”, al cual le adjudican significados que en su forma simbólica hacen referencia a la manera en que conciben a los géneros y al rol que desempeñan tanto el hombre, como la mujer, dentro de la sociedad (Figura 3).

Son varias las formas de caracterizar la lesión que denominan como "pito hembra": "El que llo- ra es la hembra y no duele. Él va creciendo, creciendo. Eso se van formando como unas téticas y comiéndole a uno, es por medio de esas téticas..., cuando uno alcanza a matar esas téticas [la enfermedad] no come más". "Como sale a los dos días empieza a comer a comer de una vez. La hembra no se profundiza va por encima y se va regando. La hembra es más fácil de curar que el macho, es la que come y hace pozo rapidito". Mientras al "pito macho" los describen de la siguiente manera: " $E l$ sale un puntito ahí y como no se moleste el demora en comer, no crece. El macho no come, él come pero para dentro, pero muy lento. Se queda como un callo, no abre la boca como la otra. No sale una bombita, sino un puntito negrito, y ahí se va creciendo. Es muy difícil de curar", así lo describió un hombre de 28 años habitante de Trigana quien tuvo la enfermedad.

El género se constituye y expresa a través de las relaciones sociales, roles y expectativas, ligados a las atribuciones femeninas y masculinas, que no son fijas y cambian según la cultura y a lo largo de la historia 19. El apelativo de macho se refiere a que es una lesión muy fuerte, más agresiva, más difícil de curar. El pito hembra se refiere a una lesión débil, menos agresiva y que responde mejor al tratamiento, así como a su capacidad de reproducción, porque se va regando.

Se denota en esta forma de referenciar la enfermedad la concepción patriarcal arraigada en el país y, en especialn en este departamento. Aunque hay una larga tradición entre las comunidades rurales de privilegiar la educación en los hijos varones 20 , al parecer son estas las que mejor manejan el concepto de la enfermedad, 
Manifestación clínica del "pito hembra" (izquierda) y del "pito macho" (derecha). Municipio de Acandí, Departamento de Chocó, Colombia, 2008.
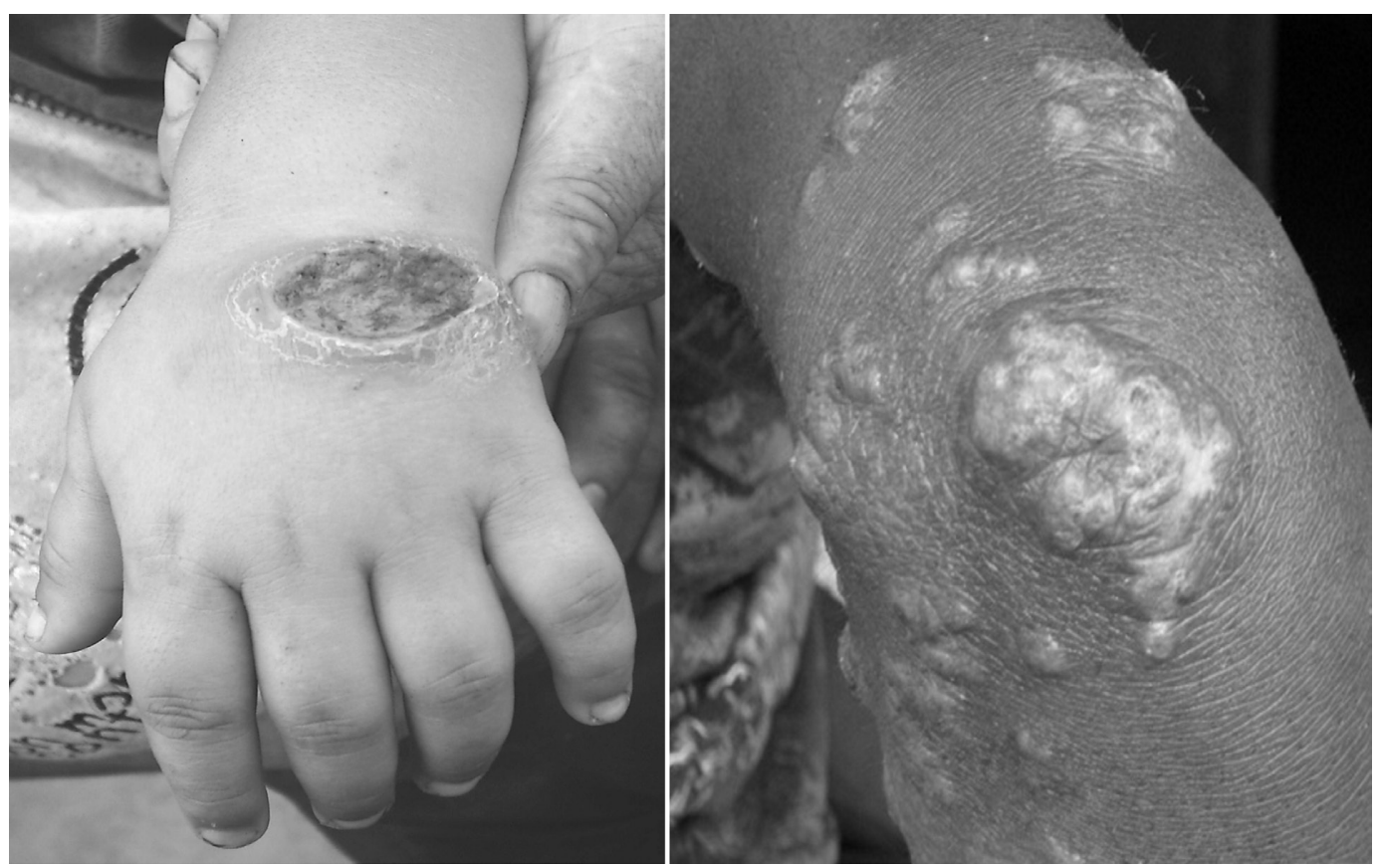

Fotógrafo: Andrés Vélez Mira.

relacionan ampliamente el pito con leishmaniasis, saben que el mecanismo de transmisión es por la picadura de un mosquito, que su tratamiento es con inyecciones, reconocen el sitio de infección en el bosque y no en la casa, y que la forma de prevenirlo es usando ropas largas. Por otra parte, los hombres reconocen el vector dentro de sus casas y al toldillo como un factor de protección.

Desde el punto de vista clínico, encontramos que la importancia que se le da a la enfermedad tiene que ver con el tipo de lesión que presentan, el lugar del cuerpo donde se manifiesta la lesión y el tiempo que lleven con ella. Si bien manifiestan cierta preocupación por el proceso físico de la lesión, existe tranquilidad ante una enfermedad que no les causa la muerte y les permite seguir trabajando.

Se encontraron diferentes conocimientos, actitudes y prácticas de acuerdo al origen de la población. Es así como los denominados paisas llegan como habitantes de la selva, pues son los sectores alejados de la cabecera de los corregimientos y zonas medianamente pobladas los preferidos por esta población para asentarse. Establecerse en un lugar hostil, como lo es el Darién para la mayoría de los citadinos, denota sacrificios y fortalezas de carácter físico y emocional para asumir el trabajo y la profunda soledad que experimentan los nuevos habitantes de estas zonas.

En este contexto, la picadura del vector, el desarrollo de la enfermedad, la cura y la subsecuente cicatriz, es un proceso con una fuerte carga simbólica. La cicatriz en la piel, es para algunos de estos migrantes una señal inequívoca de aceptación del espíritu de la naturaleza presente en ese lugar. Por esta razón, la leishmaniasis no tiene el carácter incapacitante, por el contrario, es un permiso metafísico de permanencia, llamado el "sello de la selva".

Contrariamente, para los afrodescendientes y la población "Chilapa", es una enfermedad incapacitante y desfigurante. Por esta y otras razones, estos grupos no son visitantes y mucho menos habitantes del bosque y prefieren vivir en la zona urbana de los corregimientos. 


\section{Sobre el vector}

El 80\% de los encuestados relaciona la enfermedad con la picadura de un mosquito, pero no sabe o no le proporcionan nombre. En algunas zonas como San Francisco y Triganá lo llaman "Chitra". También se conoce con el nombre de "Alus" o "Palomilla” y "Aludo". Nadie reconoce el mosquito transmisor como "Jejen".

\section{Sobre el sistema de salud}

Las condiciones del sistema de salud son precarias, el hospital de Acandí sólo cuenta con dos médicos rurales el año, quienes no tienen claro que es la leishmaniasis, ni su esquema de tratamiento. Adicionalmente, el costo del desplazamiento desde el lugar de residencia hasta el hospital oscila entre los US\$ 15 y US\$20, lo que supera la capacidad económica de algunos habitantes. En los corregimientos o veredas del estudio los centros de salud están abandonados, sólo en San Francisco hay una promotora de salud, y su puesto de salud sólo tiene capacidad para atender emergencias menores.

Hay una alta desconfianza por el sistema de salud, especialmente del que ofrece el hospital de Acandí en el diagnóstico de la leishmaniasis. Por esta razón, quienes tienen mayores recursos económicos en el sector, prefieren salir hasta Turbo para tener un diagnóstico acertado. Otros grupos económicamente menos favorecidos prefieren curarse empíricamente con la medicina tradicional antes que ir al Hospital del municipio. Toda esta suma de factores ayudan a que en la comunidad se fortalezca más la idea sobre la conveniencia de utilizar plantas u otras prácticas para cerrar las úlceras. Su pragmatismo los lleva, como último recurso, a buscar una cicatrización de la herida sin importar los medios para lograr este fin.

A pesar de lo mencionado en las encuestas, el $66 \%$ dice que acudiría al hospital si se enfermara. Sin embargo, es de resaltar que por cada hombre que se trata con un tratamiento convencional (inyecciones de antimonio de meglumine) hay 13 mujeres que lo hace de forma casera con plantas, pomadas o quemando la lesión con cuchara $\mathrm{u}$ otro metal caliente). De la misma manera, esta relación se mantiene en la decisión de recurrir al conocimiento de una persona mayor, en lugar de ir al hospital en caso de enfermarse.

Tanto los hombres, como las mujeres, están en riesgo de enfermarse, debido a la domiciliación del vector. Esto se demuestra por la inexistencia de diferencias estadísticamente significativas en la encuesta de intradermorreacción de Montenegro, que establece la prevalencia de infección (datos no mostrados) en la región. Sin embargo, las mujeres siguen sin acceder a los servicios de salud, en este caso, por su propia percepción de los sistemas de salud, como los describió Vélez et al. 21.

\section{Sobre la prevención}

Relacionan la enfermedad con ciertas prácticas que implican el acceso al bosque, como la caza o el aserrío. A la pregunta: ¿dónde cree que se adquiere la enfermedad? El $63 \%$ afirmó que en el bosque o en la selva. Esta respuesta tuvo diferencias estadísticamente significativas entre hombres y mujeres. Por cada 15 mujeres, sólo un hombre reconoce el riesgo de contraer la enfermedad en la selva. Los hombres por sus labores son la población más vulnerable en este tipo de ambiente, por lo tanto al no reconocer el riesgo en la selva, no tendrán medidas de prevención para evitar la infección en este lugar.

En contraposición sólo el 12\% afirma que en la casa se puede adquirir la enfermedad. Por cada mujer hay trece hombres que afirman que la leishmaniasis es posible contraerla en el interior de la vivienda. De la misma manera que el caso anterior, en donde parecen estar trastocados los saberes, deberían ser las mujeres quienes reconocieran este riesgo, pues son ellas las encargadas de las labores y cuidados de la familia. Al no reconocerlo como tal, se produce descuido en las medidas de prevención y control de las enfermedades vectoriales, como el uso del toldillo, las fumigaciones, entre otras. Esto puede ser un factor importante en el aumento de la prevalencia e incidencia de la enfermedad, especialmente en los niños. Por ejemplo, en San Francisco se encontró una positividad en la prueba de Montenegro en niños menores de cinco años de un 33\%.

Otro factor importante es la creencia de las personas en su capacidad de controlar lo que les pasa, o si por el contrario piensan que lo que les sucede es obra de fuerzas superiores a ellos, obra de la suerte o de una especie de dios que le pone pruebas a su fe 22 .

Este tipo de reflexiones lleva a tomar una actitud pasiva y de contemplación, con respecto a las medidas de prevención y control de la enfermedad. Si las personas creen que independiente de lo que hagan van a contraer la enfermedad, no se hará ningún esfuerzo en tomar medidas para prevenirla. Este razonamiento es reforzado y propagado por la iglesia evangélica de corte pentecostal que de algunos años para acá, más o menos siete, no sólo se ha establecido, si no que se ha propagado en la zona 16 .

Uno de nuestros interlocutores evangélicos, una mujer de 43 años argumentó: “A veces Dios 
transmite la enfermedad en una persona, para qué: para que el poder de él sea manifestado. Porque habemos [sic] muchos que decimos que Dios no hay, que Dios no existe y no es así, Dios sí existe, Dios es real, Dios tiene poder. Entonces muchas veces esas enfermedades así, son a veces permitidas por Dios para dejar ver su gloria, para que la gloria de Dios sea manifestada, sea glorificado el poder de Dios para que todo aquel que no crea, lo crea". Esto sumado al difícil acceso a los centros médicos, alarga el tiempo de curación (hasta tres años) de la lesiones, convirtiéndose en un riesgo de infección a nuevos insectos que propagarían la enfermedad 23 .

Por otro lado, a la hora de llevar a cabo sus prácticas agrícolas o desplazarse a su espacio peri- o extradomicilio que por lo general está muy cercano al bosque, lo más común es que sus pobladores no tomen ninguna medida de prevención de la enfermedad. Es habitual, por las condiciones climáticas, vestirse con prendas ligeras que no les cubren demasiado el cuerpo, lo que facilita la picadura del insecto. Por otro lado, los repelentes de insectos que serían una medida de protección son de difícil consecución y de poco acceso, debido a su costo. Finalmente, algunos habitantes del corregimiento de San Francisco de Asís consideran que la dieta a base de pescado, preparaciones a base de coco (Cocos nucifera), el siete sabores (Diospyros blancoi), y achiote (Bixa orellana L.), impiden la picadura de mosquitos y, por lo tanto, la trasmisión de la enfermedad.

\section{Sobre el tratamiento}

Todos estos factores inciden en la fuerte influencia que tienen los tratamientos no convencionales de la enfermedad. En ellos se incluyen tratamientos con plantas medicinales, el uso de moxa con tabaco y el uso de medicamentos veterinarios. Dentro de la plantas medicinales esta la que ellos nombran como Rastrera, Cadillo y Riega Plato, cuyas hojas colocan a fuego lento hasta tostarla, luego la maceran y la colocan directamente sobre la lesión como un emplasto. Pero hay particularidades en el uso, por ejemplo, con el Cadillo debe hacerse una dieta especial y no comer huevo ni yuca o cangrejos, así como no bañarse con agua salada. Y el emplasto de la última planta lo mezclan con ambramicina. Con las hojas de la planta Mata puercos hacen una infusión y con su agua, todavía tibia, la dejan caer sobre la herida. También es común el uso del tomate con antibióticos, tipo tetraciclina, así como trozos de ahuyama sobre la lesión.

Del mismo modo, la moxa de tabaco está relacionada con la eficacia que tiene el tratamiento de las lesiones con cáusticos. Esta creencia entre los pobladores radica en que la aplicación directa de calor destruye los parásitos que producen las úlceras en la piel, tal como ocurre en la termoterapia 24 . Este mismo efecto se observa en el uso de plantas calientes y emplastos, al igual que la quemadura con cuchara caliente $u$ otro metal. No hubo diferencias estadísticamente significativas entre sexo, en el uso de algunas sustancias químicas caústicas, como el ácido de batería Otras personas usan medicamentos antiparasitarios, como la ivermectina vía subcutánea en la zona abdominal en una sola dosis, la cual se repite a las dos semanas si la lesión no disminuye de tamaño. Este medicamento también es usado vía oral mezclado en agua de panela y combinado con penicilina, aplicado directamente sobre la lesión.

Estas alternativas de tratamiento, toman gran auge debido a: (1) el temor al tratamiento con antimoniales pentavalentes y sus efectos secundarios 25,26; (2) reconocen que al vivir en un área endémica, pueden enfermarse varias veces y por lo tanto no están dispuestos a someterse a este tratamiento continuamente; y (3) al hecho que el tratamiento sólo puede ser suministrado en sitios autorizados, obliga a desplazarse y abandonar sus viviendas durante el tiempo del tratamiento.

\section{Conclusión}

En el estudio se identificó que la leishmaniasis cutánea tiene diferentes connotaciones, que varían de acuerdo al origen de la población y su distribución biogeográfica. Entre las poblaciones chilapa, afrodescendiente y paisa se encontraron diferencias en los conocimientos, actitudes y prácticas frente a la enfermedad. Mientras para las dos primeras, padecer la enfermedad se convierte en problema familiar, estético y económico, para la comunidad paisa es concebida como un símbolo de bienvenida a la región del Darién y una marca de permanencia.

Por otra parte, la distribución biogeográfica de las poblaciones en especial, con respecto a su relación con el mar, a las interacciones con la población turística y al acceso a los servicios de información y de salud, marcan diferentes formas de concebir tanto la enfermedad, su denominación, así como su tratamiento y prevención. Esto se evidenció al comparar las poblaciones ubicadas en la zona costera (San Francisco de Asís, Triganá y el Aguacate) con Chugandí, ubicada en la zona montañosa.

Estos factores socioculturales nos permiten inferir que el estudio de manera fragmentada de la leishmaniasis, desconociendo el contexto 
social y cultural, circunscrito sólo en la dimensión biológica y médica, lleva al fracaso en los programas de prevención y control de la enfermedad. Este tipo de proyectos y la nueva política en ciencia y tecnología en el país, busca favorecer los estudios interdisciplinares, ayudando a abordar los problemas de investigación en forma holística.
Finalmente, el desconocimiento de la población en cuanto a las formas de transmisión, prevención y control de la enfermedad, la dificultad del acceso a la atención médica y al tratamiento evidente en la cronicidad de lesiones (de hasta tres años), así como el alto subregistro, demuestran cómo a través de una enfermedad como la leishmaniasis se desnuda el abandono del estado en esta región del país.

\section{Resumo}

A leishmaniose tegumentar americana (LTA) é considerada um problema de saúde pública em todo o mundo, e sua incidência na Colômbia é de 12 mil casos por ano. Os programas de controle implementados muitas vezes falham, provavelmente, por ignorarem as características sociais, econômicas e demográficas das regiões onde a doença ocorre. Como parte de um estudo de tipo ecoepidemiológico, neste artigo apresentamos os resultados de um estudo sobre os Conhecimentos, Atitudes e Práticas (CAP) sobre a leishmaniose em populações rurales do município de Acandí, na região do Darien colombiano. Para o estudo foram utilizados ferramentas qualitativas como a etnografia e quantitativas como levantamentos. Os resultados indicam que a população desconhece aspectos fundamentais da LTA, como por exemplo, o vetor. Além disso, existem diferenças nos CAP por sexo e tipo de população, mas não pela área geográfica. Desta forma, a nossa abordagem evidencia a negligência dos entes públicos de saúde, encabeçados pelo governo do estado. Esta negligencia é um fator determinante na perpetuação da doença.

Leishmaniose Cutânea; Conhecimentos, Atitudes e Prática em Saúde; Doenças Negligenciadas

\section{Colaboradores}

L. M. Carrillo-Bonilla realizó la investigación epidemiológica y el análisis de los datos cuantitativos en la investigación y elaboró del texto para su publicación. J. J. Trujillo realizó el trabajo de campo etnográfico, donde obtuvo los datos cualitativos y contribuyó en la elaboración del artículo. L. Álvarez-Salas participó en la construcción del artículo y la consolidación de los datos cualitativos a partir de hallazgos etnográficos recientes. I. D. Vélez-Bernal participó en el diseño de la investigación y en la supervisión del desarrollo de la misma; revisó y corrigió el artículo.

\section{Agradecimientos}

A COLCIENCIAS por la financiación de este proyecto Estudio Ecoepidemiológico para la Prevención y el Control de la Leishmaniosis en Acandí, Chocó (código 11540820520). A los investigadores Luz A. Acosta, Karina Mondragón-Shem, Andrés Vélez, Juan E. Pérez, Daniel Agudelo y Horacio Cadena del PECET por su colaboración en el trabajo de campo. A Erika T. Loaiza por el análisis estadístico. A el Grupo Biogenesis Sostentabilidad Universidad de Antioquia 2013-2014. Finalmente, agradecemos a la comunidad de Acandí su aceptación y apoyo en el desarrollo de este proyecto.

\section{Conflicto de interés}

Todos los autores declaran que no existe conflicto de interés. 


\section{Referencias}

1. World Health Organization. World health statistics 2012. http://www.who.int/gho/publications/ world_health_statistics/EN_WHS2012_Full.pdf (accedido el 10/Abr/2013).

2. World Health Organization. Control of the leishmaniases: report of a meeting of the WHO Expert Committee on the Control of Leishmaniases. Geneva: World Health Organization; 2010. (WHO Technical Report Series, 949).

3. Subdirección de Vigilancia y Control en Salud Pública, Instituto Nacional de Salud. Sistema de Vigilancia en Salud Pública - SIVIGILA. Vigilancia rutinaria, 2011. http://www.ins.gov.co/lineasde-accion/SubdireccionVigilancia/sivigila/Pagi nas/vigilancia-rutinaria.aspx (accedido el 20/Dic/ 2012).

4. Susser M, Susser E. Choosing a future for epidemiology: II. From black box to chinese boxes and ecoepidemiology. Am J Public Health 1996; 86:674-7.

5. Ariza EY, Lopez CM, Martinez O, Arias SA. Ecoepidemiología: el futuro posible de la epidemiología. Rev Fac Nac Salud Pública 2004; 22:139-45.

6. Briceño-León R. Endemic, epidemic and fashions: the sociology of health in Latin America. Revista Española de Sociología 2003; 3:69-85.

7. Bonet J. ¿Por qué es pobre el Chocó? Revista del Banco de la República 2007; 80:18-61.

8. Alta Consejería para la Reintegración, Presidencia de la República. Diagnóstico socioeconómico del Departamento del Chocó. Bogotá: Presidencia de la República; 2008.

9. Observatorio Colombiano de Ciencia y Tecnología. Indicadores departamentales de ciencia, tecnología e innovación, 2010. Chocó. http://www. ocyt.org.co/html/boletines/choco.pdf (accedido el 10/Nov/2012).

10. Organización Panamericana de la Salud. Indicadores básicos en salud. Choco 2008. http://new. paho.org/col/index.php?option=com_content\&vi ew $=$ article\&id $=770$ :indicadores-basicos-en-saludchoco-2008\&catid=687\&Itemid=361 (accedido el 04/Dic/2012).

11. Alvar J, Yactayo S, Bern C. Leishmaniasis and poverty. Trends Parasitol 2006; 22:552-7.

12. Malafaia G. Protein-energy malnutrition as a risk factor for visceral leishmaniasis: a review. Parasite Immunol 2009; 31:587-96.

13. Subdirección de Vigilancia y Control en Salud Pública, Instituto Nacional de Salud. Sistema de Vigilancia en Salud Pública -SIVIGILA. Vigilancia rutinaria, 2008. http://www.ins.gov.co/lineas-deaccion/Subdireccion-Vigilancia/sivigila/Estadisticas\%20SIVIGILA/Semana\%2049\%20de\%202008. pdf (accedido el 20/Feb/2013).

14. Salazar J. San Pacho: una tierra para la libertad. Experiencia ecológica en el Darién Caribe Colombiano. Madrid: Editorial Académica Española; 2012.
15. Álvarez L, Gálvez A. Food sovereignty in a socioecological transformation context in the Caribbean Darién of Colombia. Agroecology and Sustainable Food Systems 2014; 38:812-38.

16. Gálvez A, Salazar J, Ramírez L. Evangelical churches and conservation in San Pacho (Darien, Colombian caribbean). Universitas Humanística 2009; 68:49-67.

17. Isaza DM, Restrepo BN, Arboleda M. La leishmaniasis: conocimientos y prácticas en pobladores de la Costa del Pacífico de Colombia. Rev Panam Salud Pública 1999; 6:177-84.

18. Eco U. La estructura ausente, introducción a la semiótica. 3a Ed. Barcelona: Editorial Lumen; 1986.

19. Scott JW. Gender and the politics of history. New York: Columbia University Press; 1988.

20. Instituto Interamericano de Cooperación a la Agricultura. La situación de la mujer rural colombiana. In: Foro: Mujer Rural en Colombia: Retos y Compromisos. http://www.iica.int/Esp/regiones/andi na/colombia/Documentos\%20de\%20la\%20Ofici na/docsiica/memorias-foro-mujer-rural-2010.pdf (accedido el 28/Jul/2013).

21. Velez ID, Hendrickx E, Robledo SM, Agudelo SP. Leishmaniosis cutánea en Colombia y género. Cad Saúde Pública 2001; 17:171-80.

22. Briceño-León R. La casa enferma: sociología de la enfermedad de Chagas. Caracas: Fondo Editorial Acta Científica Venezolana/Universidad de Texas; 1990.

23. Lainson R, Shaw JJ, Ready PD, Miles MA, Póvoa M. Leishmaniasis in Brazil: XVI. Isolation and identification of Leishmania species from sandflies, wild animals and man in north Para state, with particular reference to L. braziliensis guyanensis causative agent of "pian bois". Trans R Soc Trop Med Hyg 1981; 75:530-6.

24. López L, Robayo M, Vargas M, Vélez ID. Thermotherapy. An alternative for the treatment of American cutaneous leishmaniasis. Trials 2012; 13:58.

25. Den Boer M, Argaw D, Jannin J, Alvar J. Leishmaniasis impact and treatment access. Clin Microbiol Infect 2011; 17:1471-7.

26. Marín-Leonett M. Aspectos ambientales, económicos, culturales y de comportamiento que favorecen la transmisión, y medidas de control, en la leishmaniosis tegumentaria. Rev Soc Méd-Quir Hosp Emerg Pérez de León 2007; 38:34-8.

Recibido el 30/Jun/2013

Versión final presentada el 22/Ene/2014

Aprobado el 18/Mar/2014 